\title{
A method for characterization of sea surface microlayer based on monolayer properties in presence and absence of phospholipids
}

\author{
Blaženka Gašparovića,*, Sanja Frka ${ }^{a}$, Zlatica Kozarac ${ }^{a}$, Andrew Nelson $^{b}$ \\ a Ruđer Bošković Institute, Center for Marine and Environmental Research, POB 180, HR-10002 Zagreb, Croatia \\ b Centre for Self Organising Molecular Systems, School of Chemistry, University of Leeds, LS2 9JT Leeds, UK
}

\section{A R T I C L E I N F O}

Article history:

Received 19 November 2007

Received in revised form

16 April 2008

Accepted 15 May 2008

Keywords:

Sea surface microlayer

Dioleoyl phosphatidylcholine

Model lipids

Impedance spectroscopy

ac voltammetry

Q1 Fractal analysis

\begin{abstract}
A B S T R A C T
Electrochemical impedance spectroscopy, ac voltammetry and fractal analysis were used to characterize model compounds, compound mixtures and extracted samples of sea surface microlayer (ssm) und underlying water (ulw). The reasons for carrying out this work were to use the scientific basis of these characterizations in future on-line analytical procedures of ssm. The mercury (Hg) drop electrode uncoated and coated with a monolayer of dioleoyl phosphatidylcholine (DOPC) was used as an experimental basis for investigation of the major sea surface film forming material. Firstly, the interaction of the uncoated and DOPC coated $\mathrm{Hg}$ electrode with model water insoluble compounds of increasing polarity was investigated. The compounds studied in order of increasing polarity were: nonadecane, stearic acid, cholesterol and cardiolipin. Subsequently the electrochemical response of the system to different ssm extracts was compared to signals observed with model compounds to demonstrate method selectivity. From the electrochemical results, it is observed that both the molecular structure and polarity of the investigated compounds have a role in their interaction with the uncoated and DOPC coated electrode. In the fractal analysis the increase above 2 of fractal dimension D imparted to the DOPC layer is related to the degree of apolarity of the additive model compound. Consistent with this, the more apolar hexane extracted ssm 2 imparts a fractal dimension $D$ value of 2.45 when incorporated in DOPC layers. The electrochemical response to the ssm and ulw follows that characteristic of sterol compounds.
\end{abstract}

(c) 2008 Elsevier B.V. All rights reserved. substances accumulate at the air/sea interface due to their surface-active properties. Highly apolar compounds in general represent the most important film forming material. The accumulation of hydrophilic substances in the ssm is enabled through hydrogen bonding or electrostatic interaction with the monomolecular layer [2]. Adsorbed organic substances change the physical and optical properties of the ssm. This depends on the nature of the constituent organic compounds

\footnotetext{
* Corresponding author. Tel.: +385 14561 148; fax: +3851 4680242

E-mail address: gaspar@irb.hr (B. Gašparović).
}

0003-2670/\$ - see front matter @ 2008 Elsevier B.V. All rights reserved. doi:10.1016/j.aca.2008.05.043 
which include the characteristics of the polar groups and architecture of the hydrophobic chain and seawater properties of ionic strength, $\mathrm{pH}$ and temperature.

Although the sea surface microlayers have been studied extensively, there is still a lack of knowledge about the physical chemical characteristics governing their formation and properties as well as of simple, especially in situ, methods for this characterization. The sea surface film forming material is represented by a wide spectrum of different compounds which are not soluble in water and are extractable by organic solvents. Such extracts may contain as many as 16 different subclasses of compounds of both biogenic and anthropogenic origin: aliphatic hydrocarbons, polycyclic aromatic hydrocarbons, wax esters, sterol esters, short-chain esters, acylated glyceryl ethers, triglycerides, free fatty acids, phthalate esters, free aliphatic alcohols, sterols, diglycerides, monoglycerides, glycolipids, pigments, phospholipids, arranged in an approximate order of increasing polarity [3].

Several electrochemical methods are conveniently used to characterize organic substances in sea water and the sea surface microlayer, as for example out-of-phase [4] and inphase [5-7] ac voltammetry, differential pulse anodic striping voltammetry [8], differential pulse cathodic striping voltammetry [9], and electrochemical impedance spectroscopy [10]. Up to now the fractal geometry analysis technique has not been used to characterize seawater derived organic material. The fractal geometry analysis has proved to be a useful tool in dealing with the physical and chemical problems that involve non-trivial geometries [11]. Such geometries are in fact more common in the physical world than the classical Euclidean geometries, especially if we consider surfaces and porous structures such as aggregates or fractured objects. The fractal analysis approach is based on the possibility of describing quantitatively complex objects that are statistically scale-invariant. This property is manifested in a power-law dependence of the density-density correlation function $g(r) \sim r^{D-d}$, where $D$ and $d$ denote fractal and spatial dimensions, respectively. This work therefore uses both an electrochemical impedance measurement approach combined with a fractal geometry analysis to characterize the physical chemical properties of the sea surface organic material.

The purpose of this work is to explore the possibilities of using an uncoated and a dioleoyl phosphatidylcholine (DOPC) monolayer coated mercury (Hg) drop electrode for the physicochemical characterization of sea surface forming material. The strategy is to incorporate the findings of this study into future simple electrochemical methods that may serve for the direct in situ analysis of sea surface microlayers. The DOPC monolayer was chosen as the monolayer system that can be easily prepared in a reproducible manner. In addition the fluid nature of DOPC is perfectly compatible with the smooth $\mathrm{Hg}$ surface giving a unique defect free self-sealing monolayer. Due to the different polarities of the screened compounds the interaction of each compound with DOPC is expected to vary. To demonstrate the qualitative and quantitative selectivity of the DOPC coated electrode the results from the impedance, ac voltammetry and fractal analysis for chosen model substances are compared with those observed for the sea surface microlayer extracts. This work is a continuation of preliminary studies [10] that showed that apolar hexane (Hex) extracts introduce defects and a greater surface roughness when incorporated into the DOPC monolayer due to the lower compatibility with DOPC than the polar dichloromethane (DCM) extracts. Another objective of this work was to look for a physical meaning to the fractal dimension, $D$. Although this value had been defined mathematically it seemed appropriate to measure the fractal dimension $D$ of insoluble monolayers which have already been well characterized electrochemically [12]. This paper reports experimental results for the first time where the electrochemical fractal analysis has been carried out on insoluble lipid layers.

\section{Experimental}

\subsection{Apparatus and materials}

An Autolab system, FRA and PGSTAT 30 interface (Ecochemie, Utrecht, The Netherlands), controlled with Autolab software, was used both for the electrochemical impedance spectroscopy and for the ac voltammetry. The impedance measurements were performed over a broad frequency spectrum from 65,000 to $0.1 \mathrm{~Hz}, 0.005 \mathrm{~V}$ rms at potentials of $-0.4 \mathrm{~V}$ and $1.1 \mathrm{~V}$. The impedance data were transformed to the complex capacitance plane, where the complex capacitance axes were expressed as $\operatorname{Re} Y \omega^{-1}$ and $\operatorname{Im} Y \omega^{-1}$. This plot gives a single semicircle when the capacitor has no frequency dispersion. Impedance data served to estimate the fractal dimension $D$ from the zero frequency capacitance (ZFC). The ZFC was obtained by the extrapolation of the semicircle of the complex capacitance plane to the $\operatorname{Im} Y \omega^{-1}$ axis [12]. In the ac voltammetry measurements the frequency of the ac voltage was $75 \mathrm{~Hz}$ and the amplitude $0.005 \mathrm{~V}$ rms. All experiments were performed using a three-electrode system. The working electrode was a hanging Hg drop (Metrohm, Switzerland) uncoated and coated with an adsorbed monolayer of DOPC [13], the reference electrode was $\mathrm{Ag} / \mathrm{AgCl} / 3.5 \mathrm{~mol} \mathrm{~L}^{-1} \mathrm{KCl}$ and all potentials in this paper are quoted versus this. The auxiliary electrode was a platinum rod. All measurements were carried out in the potential range from $-0.4 \mathrm{~V}$ to $-1.15 \mathrm{~V}$. The values of differential capacitance were calculated from the measured current at $90^{\circ}$ phase angle (out-of-phase). The experiments were performed in $0.1 \mathrm{M}_{\lambda} \mathrm{KCl}$ solution at $25 \pm 0.5^{\circ} \mathrm{C}$. Electrolyte $\mathrm{KCl}$ (Fisher Scientific Chemicals, Ltd.) was calcined at $600^{\circ} \mathrm{C}$. All solutions were prepared using $18.2 \mathrm{M} \Omega$ MilliQ water.

The monolayers were prepared by spreading $15 \mu \mathrm{L}$ of a $2 \mathrm{mg} \mathrm{cm}^{-3}$ working solution of ssm extract, individual model compound, compound mixture, DOPC (Lipid Products, UK), $\mathrm{DOPC}+\mathrm{ssm}$ and DOPC + ulm extract, DOPC + individual model compound and DOPC + compound mixture on deaerated electrolyte solution and then by transferring it to the Hg electrode by vertically dipping the electrode through the film [13]. The mixtures of DOPC with ssm extracts, individual model compounds and compound mixtures were prepared as weight fractions in the working solution since the molecular composition of the compounds in ssm extracts is unknown. 


\subsection{Model compounds}

The platform for the ssm characterization is based on an uncoated and a DOPC monolayer coated Hg drop electrode. DOPC is a water insoluble molecule composed of polar and apolar parts giving rise to electrostatic as well as hydrophobic reactions, respectively (Fig. 1a). For this work four model substances as representative of compounds found in sea waters were chosen [3]. Hydrocarbon: nonadecane $M_{\mathrm{W}}=268.52 \mathrm{~g} \mathrm{~mol}^{-1}$ (Aldrich), free fatty acid: stearic acid, $284.48 \mathrm{~g} / \mathrm{mol}$ (Alfa Aesar), sterol: cholesterol, $\mathrm{M}_{\mathrm{W}}=386.65 \mathrm{~g} \mathrm{~mol}^{-1}$ (MP Biomedicals) and phospholipid: cardiolipin, $M_{\mathrm{W}} \sim 1500 \mathrm{~g} \mathrm{~mol}^{-1}$ (Sigma) were used without further purification. Structural formulae are given in Fig. 1b-e. These model compounds were also chosen for their widely different polarity which is in order of increasing polarity of the compounds where nonodecane is the most apolar:nonodecane $>$ stearic acid $>$ cholesterol $>$ cardiolipin. Model compound mixtures were prepared by mixing cholesterol and cardiolipin, and cholesterol, cardiolipin and stearic acid in the weight fractions of 1:1, and 1:1:1, respectively. The molar ratios of these mixtures were $3.9: 1$ and 3.9:1:5.2, respectively. The materials were transferred to the $\mathrm{Hg}$ electrode and characterized by ac voltammetry, ac impedance and fractal analysis in the following order: (a) individual model compounds and compound mixtures, (b) DOPC + individual model compounds and DOPC+compound mixtures in specified weight fractions of: $0 \%, 17 \%, 33 \%, 50 \%$ and $66 \%$ of individual compound and compound mixture, respectively.

\subsection{Sea surface microlayer samples collection and preparation}

The sea-surface microlayer (ssm) was collected from a small eutrophic salt Lake Rogoznica connected to the mid Adriatic on August 29, 2005. Ssm 1 and ulw 1 were collected early afternoon and ssm 2 in the early evening. Sampling of the ssm was performed with a Garrett type screen made of stainless steel [14]. The thickness of the sampled microlayer was approximately 100-150 $\mu \mathrm{m}$. Ulw 1 was collected by immersing $1 \mathrm{~L}$ glass bottle at $10 \mathrm{~cm}$ depth below sample ssm 1. Natural organic material from $3 \mathrm{~L}$ of surface microlayer sample was extracted by two different solvents, hexane (HPLC grade, Merck) and dichloromethane (HPLC grade, Merck). These two solvents are of different polarities; hex is almost non-polar (0.08D) while DCM is more polar (1.14D). After three repeated extractions with solvent, the extract was evaporated to dryness in a rotary evaporator. The extracts were stored deep frozen. Prior to the analysis extracts were dissolved in the pentane and prepared to the concentration of $7.2 \mathrm{mg} \mathrm{mL}^{-1}$. The names of samples are designated for example as Hex ssm 1, where Hex is the solvent used for the extraction and 1 is the number of sample. The ulm sample was similarly extracted with DCM. The extracts were transferred to the $\mathrm{Hg}$ electrode and character-

(a)<smiles>CCCCCCCCCCCCCCCCCCCCCCCCCCCC(=O)OCC(COC(=O)CCCCCCCCCC)OC(=O)OP(=O)([O-])OCC[N+](C)(C)C</smiles>

(b)<smiles>OCCCCCCCCCCCCCCCCCCl</smiles>

(d)<smiles>CC(C)CCCC(C)C1CCC2C3CC=C4CC(O)CC[C@]4(C)C3CCC12C</smiles>

(e)

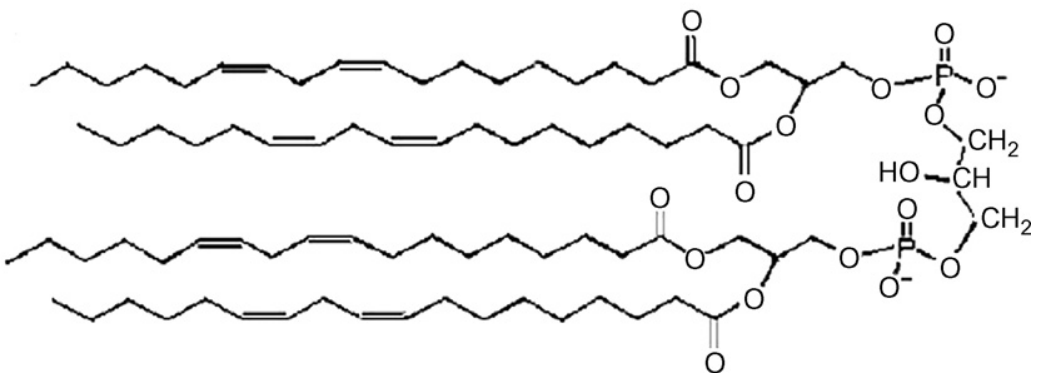

Fig. 1 - Molecular structures of dioleoyl phosphatidylcholine (DOPC) (a), nonadecane (b) stearic acid (c), cholesterol (d) and cardiolipin (e). 
ized by ac voltammetry, ac impedance and fractal analysis in the following order: (a) ssm 1, ssm 2 and ulw 1 extracts, (b) DOPC + ssm 1, DOPC + ssm 2 and DOPC + ulw 1 extracts with specified weight fractions of extract in DOPC of $0 \%, 17 \%, 33 \%$, $50 \%$ and $66 \%$.

\subsection{Fractal analysis using impedance spectroscopy data}

The adsorbed layers may exhibit fractal properties. Fractal ordering of adsorbed layers can be investigated by different techniques including electrochemical methods [15-18], atomic force microscopy [19] and transmission electron microscopy [20]. Here fractal analysis was employed with the aim of following the sensitivity of the DOPC to the ssm, ulw and model compound incorporation by changes in geometry that possesses fractal characteristics.

The fractal dimension, $D$, is determined from the slope of the log-log plot of the zero frequency capacitance (ZFC) (obtained by the extrapolation of the semicircle of the complex capacitance plane to the $\operatorname{Im} Y \omega^{-1}$ axis at low frequency [12]) versus the radius $r$ of the $\mathrm{Hg}$ drop. These results are therefore obtained from the size scaling of the hanging mercury drop electrode [15] (Fig. 2) $\left(I_{c} \sim \sim^{D}\right)$. The measurement was performed each time with a freshly prepared new drop of a selected size (the drop surface area varied from 0.0088 to $0.03815 \mathrm{~cm}^{2}$ ) that was not changed during the measurement. The reliability of the impedance data was confirmed by repetition of measurements in duplicate to check for the reproducibility and the accuracy of measurements (Fig. 2).

\section{Results}

Fig. 3 displays capacitance-potential (C-E) plots of the individual model compounds, model compound mixtures and DOPC transferred to the $\mathrm{Hg}$ electrode. The plots exhibit a flat capacity minimum at potentials close to the PZC of $\mathrm{Hg}(\sim-0.4 \mathrm{~V})$ [21], followed by capacitance increases representing phase transitions leading to desorption at more negative potentials. In the

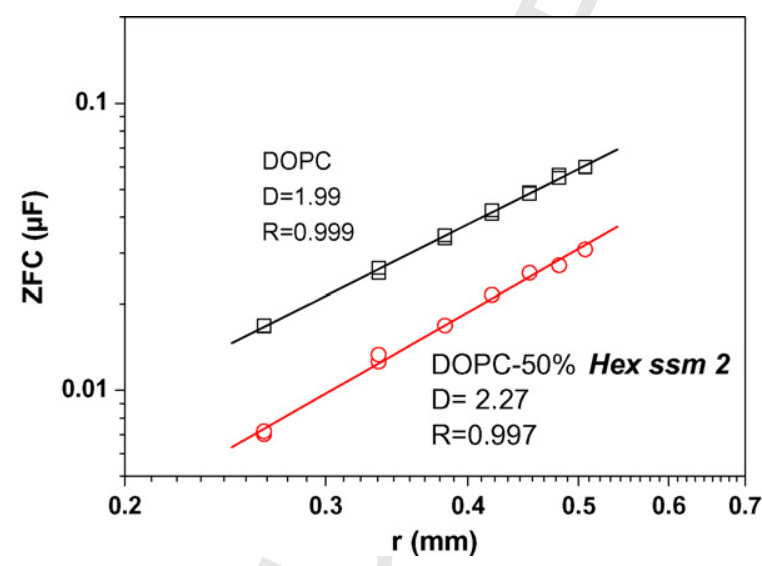

Fig. 2 - Dependence of the zero frequency capacitance on the electrode radius ( $r$ ) for DOPC $(\square)$ and DOPC $-50 \%$ Hex ssm $2(O)$ with the corresponding linear fit. $D$ and $R$ are the fractal dimension and the regression coefficient, respectively.

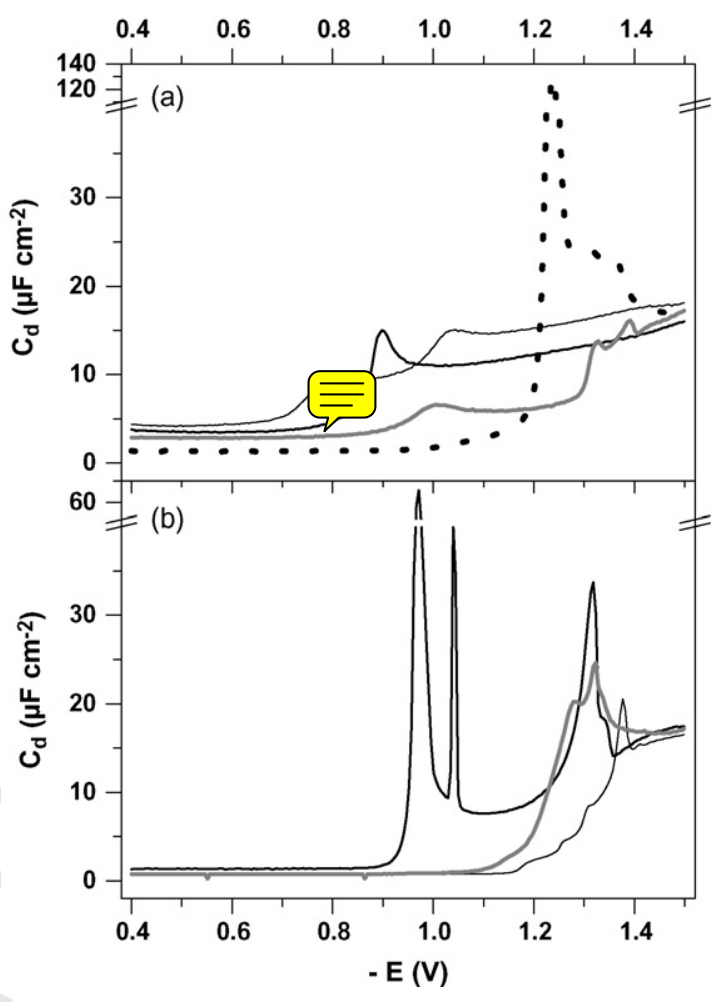

Fig. 3 - Capacitance-potential plots of (a) nonadecane (thick black line), stearic acid (thin black line), cholesterol (thick gray line) and cardiolipin (black dotted line); and (b) cholesterol + cardiolipin (1:1) (thick black line), cholesterol + cardiolipin + stearic acid (1:1:1) (thick gray line) and DOPC (thin black line).

case of the DOPC coated electrode there are two well defined capacitance peaks on the $C-E$ plot corresponding to two successive phase transitions, respectively, of the phospholipids monolayer [21]. The capacitance increases of the $C-E$ plots of the coated electrode take place at more negative potentials as the polarity of the model compounds increases (Fig. 3a). The $C-E$ plots of the mixed compound coated electrode are most similar to those of the most polar compound, cardiolipin, even though cardiolipin was present at $25 \%$ or less mole fraction in the mixture. The presence of cholesterol in the layer tends to depress and shift the capacitance peaks to more negative potentials (Fig. 3b).

In Fig. 4 are presented $C-E$ curves for the DOPC + individual model compound and DOPC + compound mixture coated electrodes. The plots show that the incorporation of cholesterol has the most marked effect on the $C-E$ curves of the DOPC monolayer coated electrode suppressing both capacitance peaks (Fig. 4c). For other mixtures transferred to the electrode the general form of the $C-E$ plots is similar to that of the pure DOPC coated electrode with a capacitance minimum and two capacitance peaks. The incorporation of the neutral hydrocarbon nonadecane in the DOPC layer shifts the DOPC capacitance peaks to less negative potentials (Fig. 4a), while the incorporation of the highly polar cardiolipin shifts the two DOPC capacitance peaks toward more negative potentials (Fig. 4d). In Fig. $4 \mathrm{~b}$ it is seen that the stearic acid added 


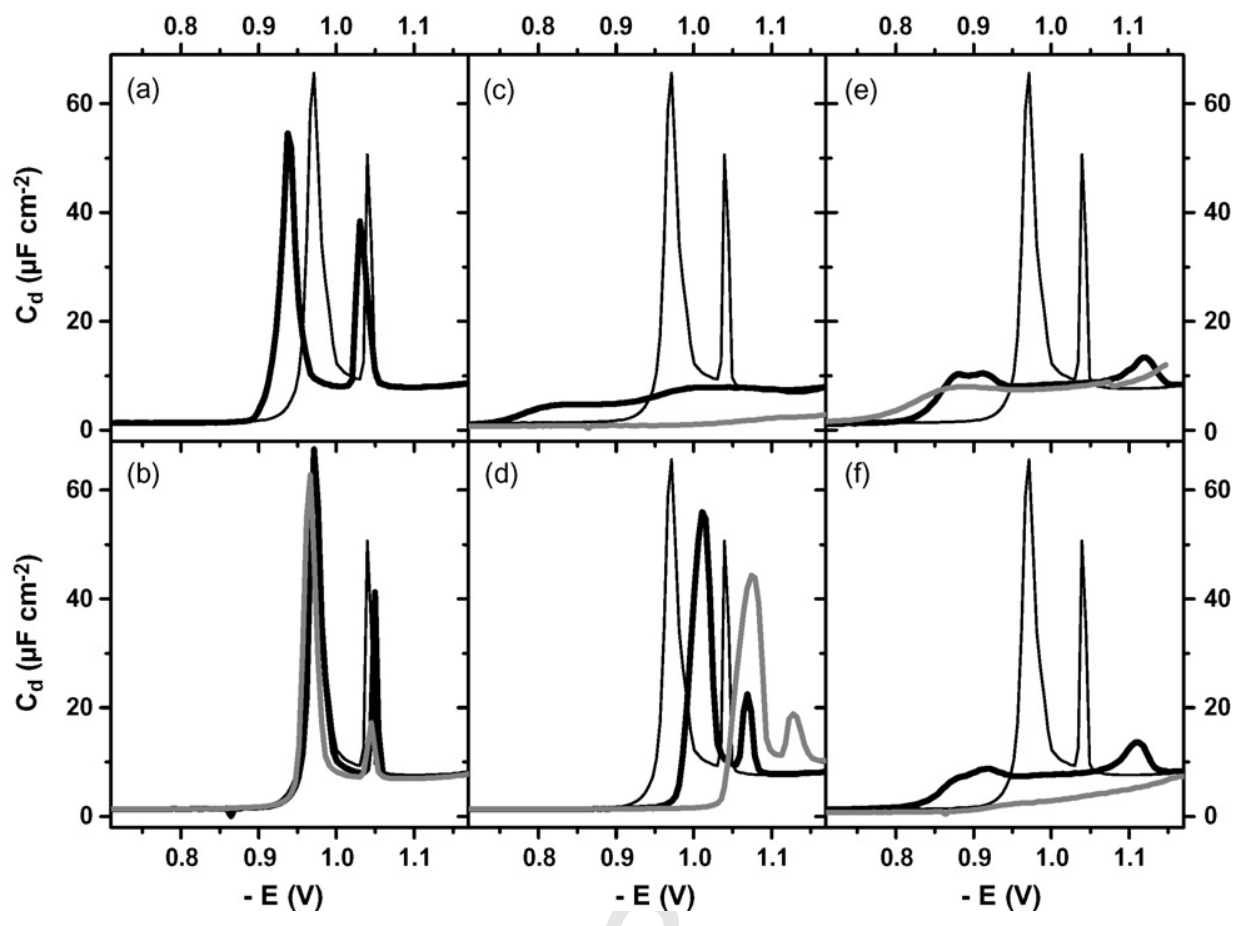

Fig. 4 - Capacitance-potential plots of DOPC (thin black line) in mixture with $17 \%$ of model substances (thick black line) and DOPC in mixture with $50 \%$ of model substances (thick gray line). DOPC with nonadecane (a), stearic acid (b), cholesterol (c), cardiolipin (d) cholesterol + cardiolipin (1: 1) (e) and with cholesterol + cardiolipin + stearic acid (1: 1: 1) (f).

to DOPC does not significantly influence the $C-E$ plot of the DOPC coated electrode. Substantial suppression of the DOPC layer capacitance peaks is observed with the mixture of DOPC and cholesterol and cardiolipin (Fig. 4e), and DOPC and cholesterol, cardiolipin and stearic acid (Fig. 4f). Fig. 5 shows changes

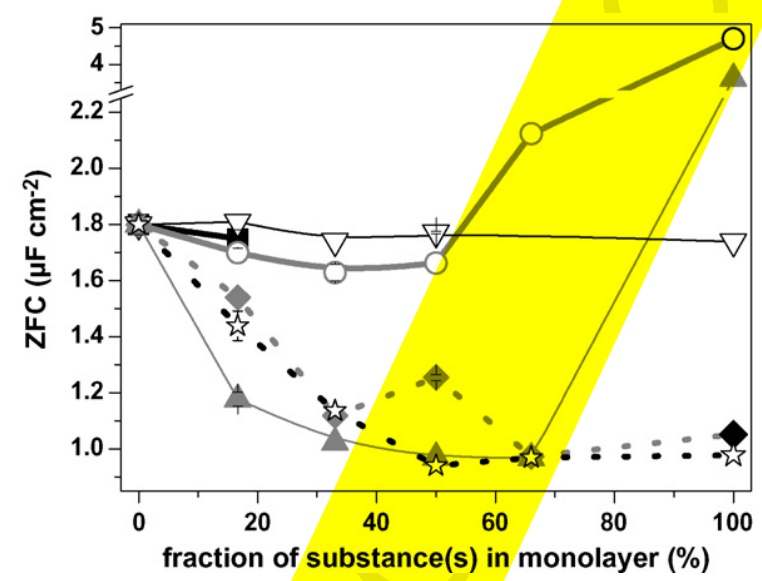

Fig. 5 - Zero frequency capacitance (ZFC) as a function of \% weight fraction of model substance(s) in DOPC; nonadecane (thick black line, black open square), stearic acid (thick gray line, gray open circle), cholesterol (thin gray line, gray full triangle), cardiolipin (thin black line, black open inverse triangle), mixture of cholesterol and cardiolipin (1:1) (gray dotted line, gray full rhombus) and mixture of stearic acid, cholesterol and cardiolipin (1:1:1) (black dotted line, black open star). Where no error bars are observed, they are within the symbol size. of zero frequency capacitance (ZFC) obtained for increasing incorporation of DOPC monolayers with the additive model compound. Only with mixtures of cholesterol and DOPC there is a significant decrease in capacitance to a minimum of $\sim 1 \mu \mathrm{F} \mathrm{cm}^{-2}$ compared to the capacitance of the pure monolayers. The ZFC plots of the DOPC and other additive compounds layers show a gradation albeit non-linear between the ZFC values of the pure compound monolayers.

Fig. 6 displays $C-E$ plots obtained from layers of Hex and DCM ssm extracts (Fig. $5 \mathrm{~F}_{\lambda}$ and $\mathrm{b}$ ) and of $50 \%$ mixtures of DOPC with Hex and DCM ssm extracts (Fig. $5 c_{\lambda}$ and d) transferred to the $\mathrm{Hg}$ electrode. $\mathrm{C}-\mathrm{E}$ plots of the Hex and DCM ssm coated electrode exhibit a capacitance minimum from $-0.4 \mathrm{~V}$ to potentials of $-0.95 \mathrm{~V}$ at which point the capacitance increases. The $C-E$ curves of the DCM ssm coated electrode (Fig. 6b) exhibit capacitance increases at more negative potentials than the $C-E$ curves of the Hex ssm coated electrode (Fig. 6a). Further the $C-E$ plot of the ssm 1 coated electrode exhibits a capacitance increase at more negative potentials than the potential characterizing the capacitance increase on the $C-E$ curve of the ssm 2 coated electrode. The $C-E$ curves of the mixtures of ssm extracts and DOPC (Fig. $6 \mathrm{~b} \hat{a n d} \mathrm{c}_{\lambda}$ are different from those of the pure extracts. Indeed, the shape of the plots is a modification of the DOPC C $-E$ plots with a capacitance minimum and two peaks and a decrease and potential shift of the two capacitance peaks which correspond to the two DOPC layer phase transitions, respectively. Notably the incorporation of the hexane extract of ssm 2 into the DOPC layer depressed the capacitance peaks on the $C-E$ plot more than the incorporation of the hexane extract of Ssm 1 (Fig. 6c). Conversely the DCM extract of ssm 1 depressed the capaci- 


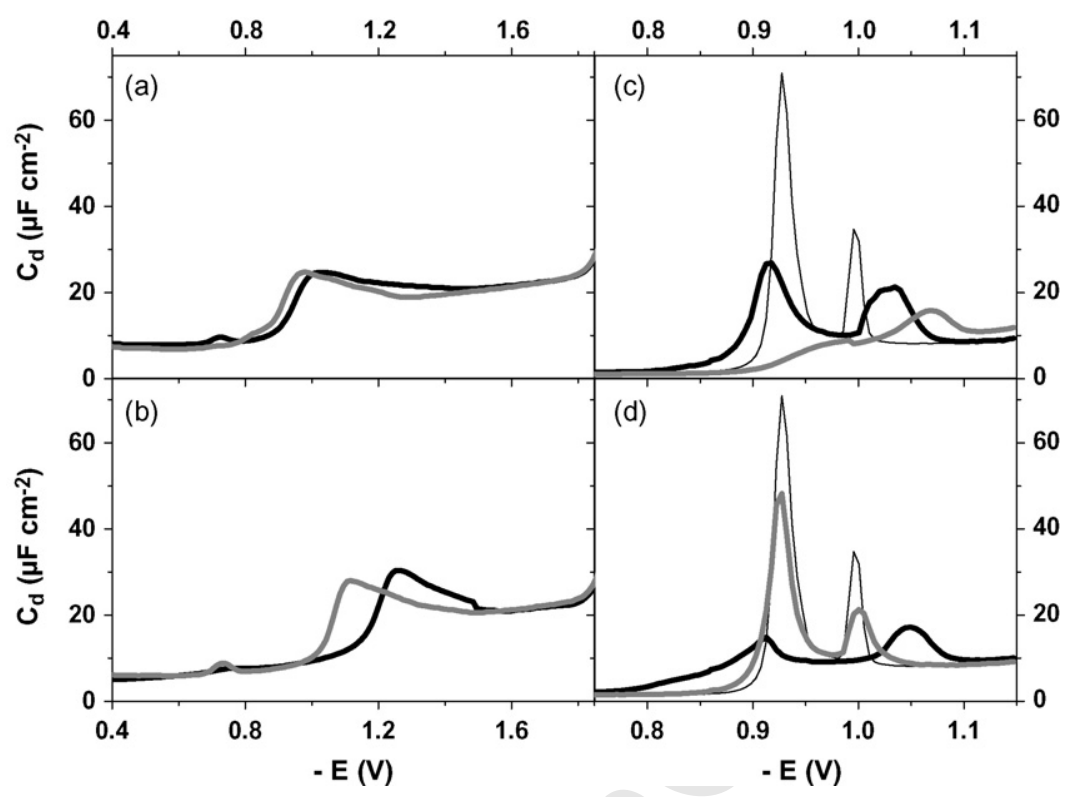

Fig. 6 - Capacitance-potential plots of (a) Hex ssm 1 (thick black line), and Hex ssm 2 (thick gray line), (b) DCM ssm 1 (thick black line), and DCM ssm 2 (thick gray line), (c) DOPC (thin black line) and DOPC in mixture with 50\% of Hex ssm 1 (thick black line) and Hex ssm 2 (thick gray line) and (d) DOPC (thin black line) and DOPC in mixture with $50 \%$ of DCM ssm 1 (thick black line) and DCM ssm 2 (thick gray line).

tance peaks on the $C-E$ plot more than the DCM extract of ssm 2. Fig. 7 shows changes of the ZFC obtained for increasing incorporation into DOPC of the ssm extracts. Increasing amounts of ssm into the monolayer results in a decrease of the ZFC compared to that of the DOPC monolayer coated electrode. The DOPC + Hex ssm 2 layers showed a lower ZFC than the DOPC + Hex ssm 1 layers whereas the DOPC + DCM ssm 1 layers showed a lower ZFC than the DOPC + Hex ssm 1 layers. Note also that the DCM extract of the underlying water below ssm 1 (ulw 1) when added to the DOPC depresses the ZFC even further.

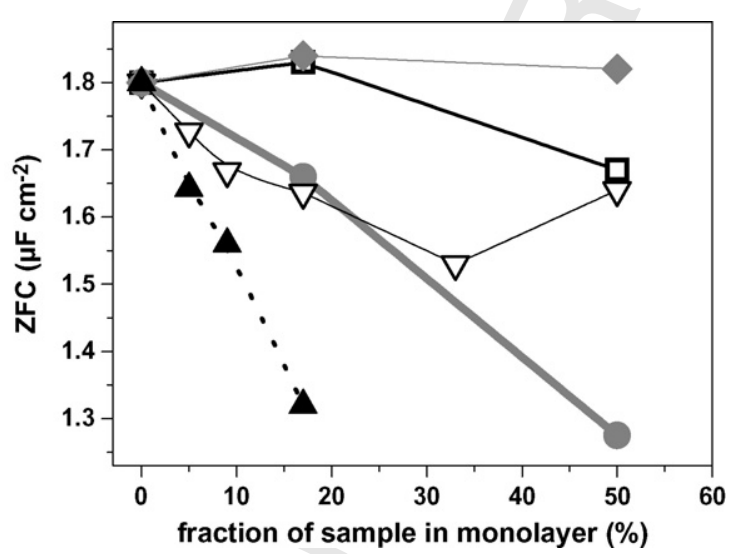

Fig. 7 - Zero frequency capacitance (ZFC) as a function of $\%$ weight fraction of Hex ssm 1 (thick black line, black open square), Hex ssm 2 (thick gray line, gray full circle), DCM ssm 1 (thin black line, black open inverse triangle), DCM ssm 2 (thin gray line, gray full rhombus) and DCM ulw 1 (black dotted line, black closed triangle) in DOPC.
Fig. 8 shows changes of fractal dimension $D$ for increasing weight fraction of individual model compounds (Fig. 8a), model compound mixtures (Fig. $8 \mathrm{~b}$ ) and the ssm extracts (Fig. 8c), respectively, in the DOPC monolayer. Interaction of different compounds with DOPC is manifested as a significant change of the adsorbed layer fractal dimension $D$. It is noted that the DOPC monolayer at $-0.4 \mathrm{~V}$ has no fractal ordering and its dimension $D$ is $\sim 2$ similar to the uncoated Hg surface. On the other hand, the fractal dimension $D$ of the monolayer mixture with model substances of different polarity increases with decreasing polarity of model lipid. For the case of compound mixtures, the changes of $D$ show more complex behaviour (Fig. 8b) with $D$ generally being above 2 although in one case lower than 2 for the more polar mixture. The mixtures of the ssm extracts with the DOPC monolayer produce layers which in certain situations exhibits fractal ordering. For the two ssm samples each extracted by two solvents, nonpolar Hex and the more polar DCM, the fractal dimension of monolayer is in the main lower than 2. The exception is the DOPC + Hex ssm 2 layer which exhibited a fractal dimension D of 2.45 .

\section{Discussion}

The increased stability of the more polar compounds and extracts on the $\mathrm{Hg}$ with respect to potential domain is intuitively to be expected. Clearly at more polar negative potentials the less polar compounds and material will have a lower stability on the electrode.

The interaction between DOPC and the model compounds characterized by the C-E plots is dominated by the structural features and polarity of the compounds. Out of all the compounds investigated, cholesterol has the strongest influ- 


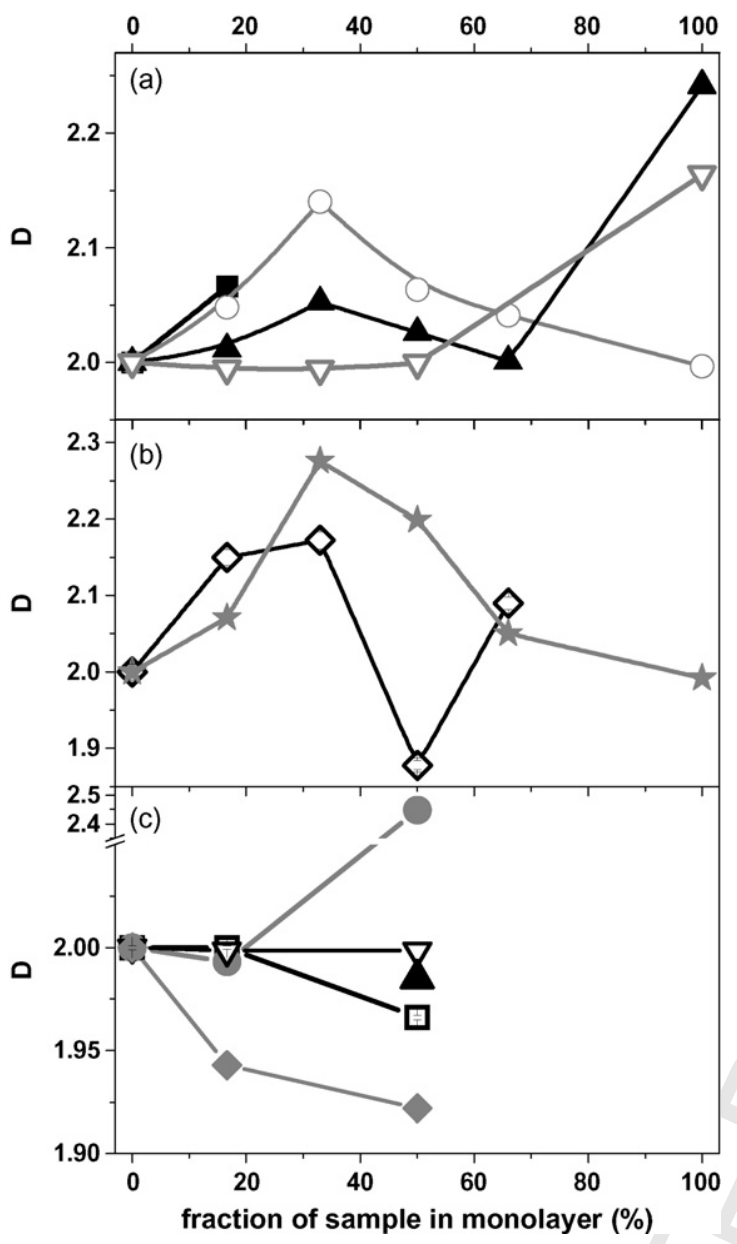

Fig. 8 - Fractal dimension (D) determined for increasing weight fraction of model or sample in DOPC monolayer for (a) nonadecane (thick black line, black closed square), stearic acid (thin gray line, gray open circle), cholesterol (thin black line, black closed triangle), cardiolipin (thick gray line, gray open inverse triangle); (b) cholesterol + cardiolipin (1:1) (thin black line, black open rhombus), cholesterol + cardiolipin + stearic acid (1:1:1) (thin gray line, gray full star); and (c) Hex ssm 1 (thick black line, black open square), Hex ssm 2 (thick gray line, gray full circle), DCM ssm 1 (thin black line, black open inverse triangle), DCM ssm 2 (thin gray line, gray closed rhombus) and DCM ulw $1(7)_{1}$ Where no error bars are observed, they are within the symbol size.

a capacitance of $\sim 4 \mu \mathrm{F} \mathrm{cm}^{-2}$ and a fractal dimension $D$ of 2.3 . This accords with the different properties of pure cholesterol compared to DOPC + cholesterol monolayers. Pure cholesterol monolayers when deposited on self-assembled monolayers of 2-naphthalenethiol (2-NT) and thiophenol (TP) have been shown to be tilted at $26^{\circ}$ from the normal. Capacitance values of these layers were similar to those observed in this study and AFM and STM studies showed that the cholesterol layers were structured with striped domains [23].

One of the aims of this work was to look for a relation between the fractal dimension $D$ and the value of $\beta$ extracted from the impedance versus frequency data using the impedance model developed in the paper by Whitehouse et al. [24] and applied to numerous experimental situations (e.g. [10]). $\beta$ has been proposed to be an indicator of departures from ideality at the interface between the monolayer surface and the bulk electrolyte which relate to a surface roughness. When $\beta=1$ the monolayer surface is smooth and when $\beta$ is $<1$, the monolayer develops a roughness. Normally a DOPC layer on mercury gives a $\beta$ value of 0.998 but following the adsorption of peptides [24] and the binding of proteins [25] this value is decreased to $0.990-0.980$. Analysis of the impedance versus frequency plots in this study showed that DOPC + cholesterol layers always exhibited a $\beta$ value of 0.998 . On the other hand the pure cholesterol layer on $\mathrm{Hg}$ showed a lower $\beta$ value of 0.990. Experimental errors for $\beta$ determination vary from sample to sample and were less than 0.001 . This therefore accords with the very different properties of the pure cholesterol monolayer for which fractal analysis evidence shows them to be structured.

Cardiolipin monolayers show similar properties to those of DOPC in terms of the $C-E$ curve although as pure monolayers they exhibit a fractal dimension value D of 2.15. Cardiolipin carries two negative charges and incorporates charged groups to the monolayer surface. The charged groups might impart some structure to the pure cardiolipin monolayers. Cardiolipin has already been shown to strongly influence the thermodynamic properties of monolayers and bilayers of egg phosphatidylcholine (egg PC) [26]. Cardiolipin also stabilises membranes of egg PC [27]. In this study the effect of cardiolipin is to alter the energetics of the DOPC phase transitions which are shifted to more negative potentials. Stearic acid $\left(\mathrm{pK} \mathrm{K}_{\mathrm{a}}=10.15\right.$ [28]) is neutral at the $\mathrm{pH}$ used in this study and the interaction with the polar head groups is through the $-\mathrm{COOH}$ group. Indeed stearic acid has been reported as forming a 2:1 molar ratio complex with PC which occupies more area than the three individual moles [29]. If this complex forms on the $\mathrm{Hg}$, this study shows that it has little effect on the capacitative properties of the layer. In all cases where either mixtures of model compounds or ssm extracts with DOPC are studied, the desorption potentials at $C-E$ curves indicate that the compounds/extracts are associated with the DOPC and not adsorbed on the Hg.

Comparing the extracts of the ssm 1 and ssm 2 it may be concluded that the ssm 1 extract was characterized by the presence of more polar compounds than the ssm 2 extract. Evidence to support this came from the following: (a) Desorption peaks on $C-E$ curve of ssm 1 extract on $\mathrm{Hg}$ are shifted toward more negative potentials than desorption peaks of $C-E$ curve of the extract of ssm 2 on Hg. (b) The DCM extract from ssm 
1 when incorporated in the DOPC monolayer depressed the capacitance peaks of the $C-E$ curve (of the mixed monolayer) to a greater extent than the hexane extract incorporation. The decrease in ZFC of the DOPC monolayer and the depression of the capacitance peaks on the $C-E$ plots with the DCM extract of ssm 1 and in particular of DCM extract of ulw 1 indicate a significant contribution of sterol material that is associated with phytoplankton living organisms. Sterols share with phospholipids a structural function in membranes acting as specific internal regulators of membrane fluidity and influencing various membrane functions [30]. It has to be expected that there are higher contribution of sterols in underlying water samples as compared to microlayer samples for afternoon sample 1. This is due to the escape of phytoplankton from strong sun irradiation at the microlayer during sampling period. This shows that firstly the technique used distinguishes between the ssm and the ulw and secondly that the ulw contained more sterol type material than the overlying ssm.

Fractal analysis is a powerful characterization tool since the fractal dimension is sensitive to subtle structural change(s) of the adsorbed layer including layer saturation, isotropic to nematic transition, and orientational change of adsorbed molecules [16]. Conceptually a value of $D$ above or below 2 means the capacitance does not increase as a linear function of electrode area. If $D$ is greater than $2\left(C \sim r^{2-3}\right)$ there is a part volume element to the capacitance value and if $D$ is less than $2\left(C \sim r^{1-2}\right)$ there is a part onedimensional element to the capacitance value. The physical meaning attributed to this [31] is that this effect is introduced from a structured inhomogeneity of the monolayer leading to an uneven distribution of dielectric, dielectric thickness and distribution of charges on the monolayer surface.

The tendency for $D$ to be $>2$ in the case of more apolar compounds being incorporated in the DOPC monolayer (see Fig. 8(a)) may be related to phase separation between the polar DOPC and the apolar compound additive leading to some structural inhomogeneity. Cholesterol is well known to separate into two domains in mixtures with phospholipids [32] and this phase separation is detected by a $D$ value of 2.05 . This would accord also with $D>2$ when the compound mixtures are incorporated into the DOPC monolayer (see Fig. 8(b)). In the case of such complex mixed monolayers some phase separation is inevitable. A value of $D<2$ is also observed for these mixtures. We conclude that for homogeneous mixed monolayers, the fractal dimension $D$ tends to the value of 2 . Where phase separation occurs as when apolar compounds or more complex compound mixtures are incorporated into DOPC monolayers, the fractal dimension $D$ generally goes above 2 . In cases where the fractal dimension $D$ goes below 2 this relates also to phase separation since it is generally observed in complex lipid mixtures and in mixtures of the more polar ssm with DOPC (see Fig. 8(c)).

The layer derived from the extract Hex ssm 2 when incorporated into a DOPC layer exhibited a very high value of fractal dimension, $D=2.45$. This value would be expected from the results of the mixed model compound-DOPC layers where it has been concluded that incorporating apolar compounds into a DOPC monolayer effected some phase separation. We cannot at this stage comment on the precise physical signifi- cance of the $D$ value being $>2$ or $<2$ but we can conclude that the value of $D$ is a sensitive indicator of the homogeneity of the monolayer which correlates with a phase separation of domains.

\section{Conclusions}

This paper has developed a scientific basis for applying capacitative electrochemical methods to the analysis of the sea surface microlayer. Work in development in our laboratories to be published later will use more robust electrode supports configured on an on-line platform. The results obtained in this study will enable results from these on-line devices to be interpreted and related to the composition of the sea surface microlayer. The findings of this study of direct relevance to this future analytical technology can therefore be summarised in the following:

(i) The adsorption of a series of insoluble compounds (nonadecane, stearic acid, cholesterol, cardiolipin) on $\mathrm{Hg}$ showed that the polarity of the compounds determined their stability on the electrode with respect to applied potential. The most polar compounds were stable over the widest potential domain.

(ii) The capacitative properties of mixed monolayers of insoluble compounds with DOPC were governed by those of DOPC. Cholesterol decreases the fluidity of the monolayer suppressing the phase transitions and increasing the monolayer thickness whereas cardiolipin affected the energetics of the phase transitions by shifting them to more negative potentials. The other compounds had only small effects.

(iii) Studies of the fractal dimension of mixtures of insoluble compounds and DOPC carried out for the first time showed that the extent of the fractal dimension $D$ imparted to the DOPC by the compound depended on its non-polarity. The more apolar compounds (stearic acid, nonadecane) imparted the higher value of fractal dimension $D$. Pure cholesterol monolayers were distinct in showing a fractal dimension $D>2$ whereas mixed cholesterol-DOPC layers showed a fractal dimension $D$ of 2 . More complex mixtures with DOPC and mixtures of polar ssm with DOPC showed fractal dimension $D$ values of $<2$.

(iv) The ssm 2 sample was dominated by non-polar material and the ssm 1 sample by polar material. The effects of the sample extracts on the DOPC monolayer capacitive properties resembled those of cholesterol or sterols in general.

(v) In mixed monolayers the origin of the fractal dimension $D$ values of above and below 2 could be related to phase segregation of the additive compounds from the DOPC.

The findings show that the approach can be used to identify major lipid classes in the sea surface microlayer. It has the potential of being a very powerful preliminary screening analytical method when used in an on-line configuration. The methodology allows us to conclude on the dominant physical characteristics of compounds, whether polar or apolar interact within a monolayer on the electrode surface. 


\section{Acknowledgements}

The authors acknowledge support from The Royal Society (UK) International Joint Project Program and the Croatian Ministry of Science, Education and Sport, Project "Nature of organic matter, interaction with traces and surfaces in environment" (No. 098-0982934-2717).

\section{REFERENCES}

[1] P.S. Liss, R.A. Duce (Eds.), The Sea Surface and Global Change, University Press, Cambridge, 1997.

[2] Z. Kozarac, B. Ćosović, D. Mobius, M. Dobrić, J. Colloid Interface Sci. 226 (2000) 210.

[3] C.C. Parrish, T.A. Abrajano, S.M. Budge, R.J. Helleur, E.D. Hudson, K. Pulchan, C. Ramos, in: P. Wangersky (Ed.), Lipid and Phenolic Biomarkers in Marine Ecosystems: Analysis and Applications (Handbook of Environmental Chemistry, Marîne Chemistry 5 (Part D)), Springer, Berlin, 2000, pp. 193-223.

[4] B. Ćosović, V. Vojvodić, Limnol. Oceanogr. 27 (1982) 361-369.

[5] B. Gašparović, B. Ćosović, Mar. Chem. 75 (2001) 301.

[6] T. Zvonarić, V. Žutić, M. Branica, Thalassia Jugosl. 9 (1973) 65

[7] M. Plavšić, B. Gašparović, B. Ćosović, Fresen. Environ. Bull. 16 (2007) 372.

[8] M. Plavšić, D. Krznarić, M. Branica, Mar. Chem. 11 (1982) 17.

[9] M. Plavšić, X.K. Lu, C.M.G. Van Den Berg, Croat. Chem. Acta 70 (1997) 179.

[10] S. Frka, A. Nelson, Z. Kozarac, Int. J. Environ. Anal. Chem. 86 (2006) 325.

[11] B.B. Mandelbrot, The Fractal Geometry of Nature, Freeman, San Francisco, 1982.

[12] C. Whitehouse, R. O'Flanagan, B. Lindholm-Sethson, B. Movaghar, A. Nelson, Langmuir 20 (2004) 136.
[13] A. Nelson, A. Benton, J. Electroanal. Chem. 202 (1986) 253.

[14] W.D. Garrett, Limnol. Oceanogr. 10 (1965) 602.

[15] D. Risović, B. Gašparović, B. Ćosović, Langmuir 17 (2001) 1088.

[16] B. Gašparović, D. Risović, B. Ćosović, Electrochim. Acta 49 (2004) 3383.

[17] M. Hromadova, R. Sokolova, L. Pospišil, N.J. Fanelli, Phys. Chem. B 110 (2006) 4869.

[18] B. Gašparović, D. Risović, B. Ćosović, A. Nelson, Electrochim. Acta 52 (2007) 2527.

[19] F.L. Leite, L.G. Paterno, C.E. Borato, P.S.P. Herrmann, O.N. Oliveira, L.H.C. Mattoso, Polymer 46 (2005) 12503.

[20] H. Nygren, Biophys. J. 65 (1993) 1508.

[21] D. Bizzotto, A. Nelson, Langmuir 14 (1998) 6269.

[22] A. Radhakrishnan, H.M. McConnell, Biophys. J. 77 (1999) 1507.

[23] R.K. Pandey, K.A. Suresh, V. Lakshminarayanan, J. Colloid Interface Sci. 315 (2007) 528.

[24] C. Whitehouse, D. Gidalevitz, M. Cahuzac, R.E. Koeppe II, A. Nelson, Langmuir 20 (2004) 9291.

[25] S. Weiss, P. Millner, A. Nelson, Electrochim. Acta 50 (2005) 4248.

[26] S. Nichols-Smith, S.-Y. Teh, T.L. Kuhl, Biochim. Biophys. Acta 1663 (2004) 82.

[27] P.R. Allegrini, G. Pluschke, J. Seelig, Biochemistry 23 (1984) 6452.

[28] J.R. Kanicky, O.D. Shah, J. Colloid Interface Sci. 256 (2002) 201.

[29] A.D. Petelska, M. Naumowicz, Z.A. Figaszewski, Bioelectrochemistry 70 (2007) 28.

[30] Z.A. Wojciechowski, in: G.W. Patterson, W.D. Nes (Eds.), Physiology and Biogeochemistry of Sterols, American Oil Chemists' Sociêty, Champaign, IL, 1991, p. 361.

[31] D. Risović, B. Gašparović, B. Ćosović, J. Phys. Chem., B 106 (2002) 9810.

[32] H.M. McConnell, M. Vrljić, Annu. Rev. Biophys. Biomol. Struct. 32 (2003) 469. 